\title{
Laser-Assisted Telerobotic Control for Enhancing Manipulation Capabilities of Persons with Disabilities
}

\author{
Karan Khokar, Kyle B. Reed, Redwan Alqasemi and Rajiv Dubey
}

\begin{abstract}
In this paper, we demonstrate the use of range information from a laser sensor mounted on the end-effector of a remote robot manipulator to assist persons with limited upper body strength carry out Activities of Daily Living in unstructured environments. Laser range data is used to determine goals, identify targets, obstacles, and via points to enable autonomous execution of trajectories. The human operator is primarily involved in higher level decision making; the human user performs minimal teleoperation to identify critical points in the workspace with the laser pointer. Tests on ten healthy human subjects in executing a pick-and-place task showed that laser-based assistance not only increased the speed of task execution by an average of $26.9 \%$ while decreasing the physical effort by an average of $85.4 \%$, but also made the task cognitively easier for the user to execute.
\end{abstract}

\section{INTRODUCTION}

According to the 2006 US Census Bureau report [1] 51.2 million Americans suffer from some form of disability and 10.7 million of them are unable to independently perform activities of daily living (ADL). They need personal assistance to do ADLs such as to pick-and-place an object or open a door. Robotic devices have been used to enable physically disabled individuals to execute ADLs [2]. However, teleoperation of a remote manipulator puts a lot of physical and cognitive load on the operator [2] more so for persons with disabilities. There have been previous attempts to provide computer based assistance by combining teleoperation and autonomous modes in shared and traded control formulations [3] [4] [5], by means of virtual fixtures [6] and potential fields [7]. Previous work at the Rehabilitation Robotics Laboratory at the University of South Florida has focused on reducing operators fatigue by providing assistance depending on the accuracy of sensor and model information [8], augmenting the performance of motionimpaired users in job-related tasks using scaled teleoperation and haptics [9], and providing assistance based on real-time environmental information and user intention [10]. In this work we use the laser sensor to minimize the physical and mental burden on the human user during task execution. The laser range data is used to determine goal points and identify

Manuscript received March 10, 2010. Final Mauscript Received July 15, 2010. This work was supported by the NSF Grant No. IIS-0713650. Karan Khokar is at the University of South Florida, Tampa, FL 33620 USA (phone: 813-447-7703; e-mail: karan.khokaregmail.com)

B. Reed is at the University of South Florida, Tampa, FL 33620 USA (phone: 813-974-2385; e-mail: kylereedeusf.edu)

Redwan Alqasemi is at the University of South Florida, Tampa, FL 33620

USA. (e-mail: alqasemi@eng.usf.edu)

Rajiv Dubey is at the University of South Florida, Tampa, FL 33620 USA.(e-mail: dubey@eng.usf.edu) targets, obstacles, and via points in the remote unstructured environment that enables autonomous execution of certain subtasks under human supervisory control, thus providing assistance to the human user. The human is still in the loop and teleoperates to point the laser to critical points in the remote environment. The authors believe that the use of the laser range information in this manner is a novel approach. The motivating factor behind this work is to enable persons with limited upper body strength (due to multiple sclerosis, muscular dystrophy, heart stroke or spinal cord injuries) to execute ADLs. However, the proposed telerobotic concept has a much broader scope in terms of providing assistance in areas such as nuclear waste clean-up, space/undersea telerobotics, robotic surgery, and defense applications.

\section{RELATED WORK}

Hasegawa et al. [11] enabled autonomous execution of tasks by generating 3D models of objects with a laser sensor that computed 3D coordinates of points on objects. These models were compared to a database of CAD models to match objects. Takahashi and Yashige [12] presented a simple and easy to use laser-based robot positioning system to assist the elderly in doing daily pick-and-place activities. The robot in this case was an $x-y-z$ linearly actuated mechanism mounted on the ceiling. Nguyen et al. [13] made use of a system consisting of a laser pointer, a monochrome camera, a color filter and a stereo camera pair to estimate the 3D coordinates of a point in the environment so their robot could fetch objects in the environment designated with the laser pointer. The methodology that we present for task execution is simple and uses a single point laser sensor. The information necessary to enable task execution is generated quickly online. Moreover the interface is easy to use, which is necessary in assistive robotics for persons with disabilities.

\section{LASER-ASSISTED CONTROL CONCEPT}

The human user teleoperates a PUMA manipulator via a Phantom Omni haptic device. First, the user points the laser mounted on the PUMA end-effector to critical points in the environment by teleoperating. These critical points could be goal points, objects, or planar surfaces of interest. Referring to Fig. 1, the laser sensor is mounted on the PUMA endeffector so the laser beam direction will always be parallel to the $z$-axis of the end-effector. Thus, by teleoperating the PUMA wrist (i.e., joints four, five, and six) the user is able to access a major portion of the PUMA workspace with the laser pointer. 


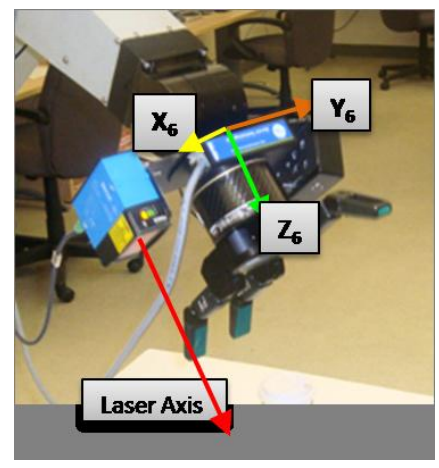

Fig. 1. Laser sensor mounted on the end-effector.

\section{A. Laser-Assisted Target Position Determination and Au- tonomous Trajectory Execution}

As the user points the laser to a specific object and presses a keyboard key, the system generates a linear trajectory from the current PUMA end-effector location to that point on the object and executes the trajectory. In an actual implementation for a physically disabled individual, the keyboard inputs would be large buttons serving the same functions.

For generating a linear trajectory, the initial point transformation matrix with respect to the PUMA base is determined from the PUMA forward kinematics and that for the final point is determined as follows. Since the orientation of the laser and the PUMA end-effector frames remain the same at all times and if we let the orientation of the end-effector remain the same at the beginning and at the end of the trajectory then the only unknown in the determination of the final point transformation matrix is the distance of the selected point from the laser sensor, $D$, which is determined from the laser range data. Referring to Fig. 2 and using 1 below, we are able to determine the target point transformation matrix.

$$
T_{O}^{B}=T_{E}^{B} * T_{L}^{E} * T_{O}^{L}
$$

The trajectory, in the form of via points ${ }^{\prime}$ transformation matrices, is generated using linear interpolation and the equivalent angle-axis method. After via points are computed and stored in an array, they are read at a rate of $200 \mathrm{~Hz}$ and joint angles are determined from these using the Resolved Rate algorithm. These angles are fed to the torque generator at the same rate to compute torque using a PD control law. This causes the manipulator to traverse the trajectory autonomously.

\section{B. Laser-Assisted Planar Surface Orientation Determination and Autonomous Surface Alignment}

For certain ADLs, determining the orientation of the planar surface associated with the target is necessary to orient the end-effector in such a way that it is aligned with the surface normal. This helps the user manipulate a target from a convenient angle during teleoperation. This feature has been implemented as an autonomous function using laser data. In a door-opening task, aligning the gripper with the door surface puts the arm in a convenient configuration to manipulate the

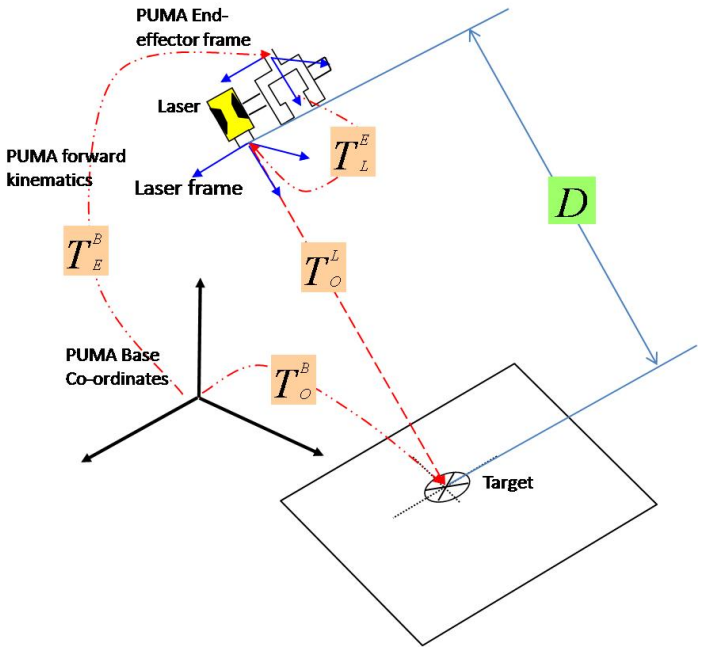

Fig. 2. Autonomous trajectory generation concept.

door handle whereas in a pick-and-place task the autonomous alignment puts the arm in a convenient configuration to grasp an object.

The user specifies a plane by pointing the laser to three distinct points on the planar surface. Using the method adopted in the previous section, the transformation matrices of these points with respect to the PUMA base are calculated. The translation component of the transformation matrix gives the coordinates of those points. The points are shown in Fig. 3 as $P_{1}, P_{2}$, and $P_{3}$. Later in the task execution, when autonomous alignment is needed, the user hits a keyboard key. On doing so, the transformation matrix that the endeffector has to attain for alignment is computed online and the end-effector aligns with the surface. The Resolved Rate algorithm has been used for autonomous rotation. Since the end-effector is rotating at the same location in space, its transformation matrix for alignment will have the same translation vector and the rotation matrix is determined as follows. The $z$-component of the rotation matrix is the same as the unit vector along the surface normal, which is computed by the cross product of the vectors connecting the three points $P_{1}, P_{2}$, and $P_{3} . \vec{C}$ is the surface normal (Fig. 3) that computes as $\vec{A} \times \vec{B}$. The $x$ and $y$-components are then obtained using the right-hand rule.

\section{ApPlication Of Laser-Assisted CONCEPT IN TASK EXECUTION}

Here we give examples of two ADLs that can be executed in unstructured remote environments using laser-based capabilities and demonstrate how it can be used to assist the user in executing these tasks.

\section{A. Pick-and-Place Task Execution}

In a pick-and-place task, the remote robot commanded by the user picks up a target object from a source point and places it at the destination point while avoiding obstacles. The user starts with locating the destination point by pointing the laser in teleoperation and commanding the system to 


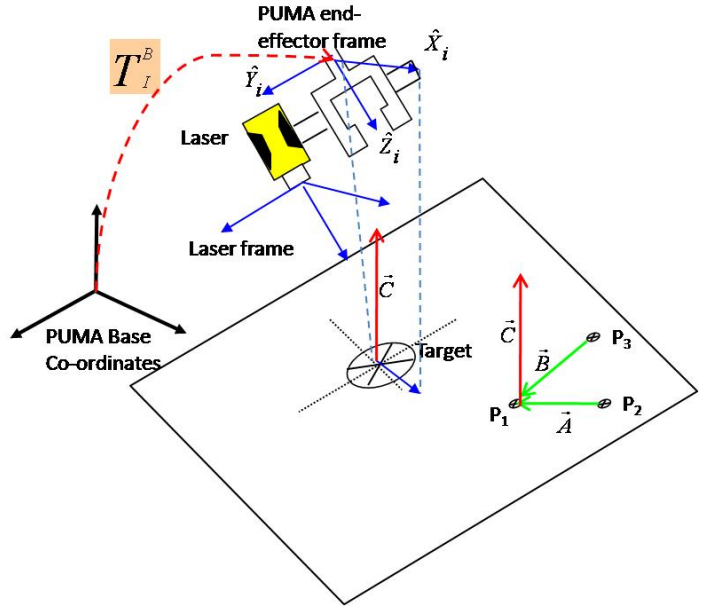

(a) Before alignment

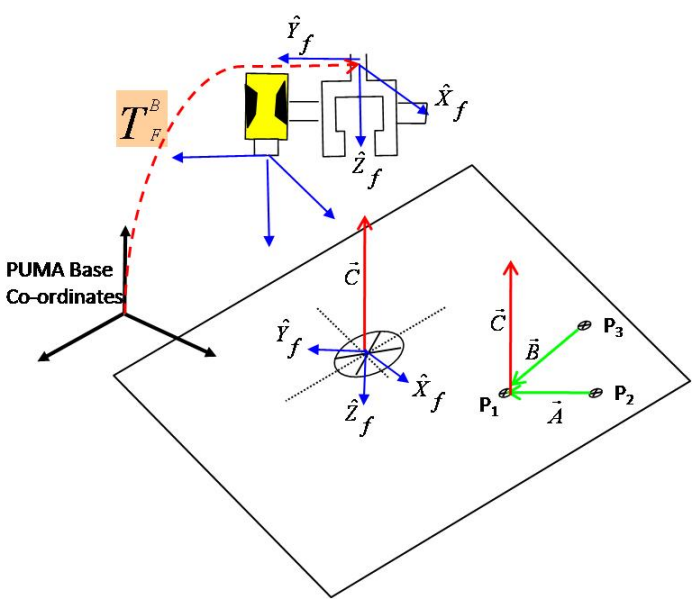

(b) After alignment

Fig. 3. End-effector configurations in autonomous surface alignment.

record the coordinates of that point (Fig. 4(a)). The user then points the laser to the top of the tallest obstacle in the remote environment to record the coordinates of the top-most point of the obstacle. This information is sufficient for obstacle avoidance for the path the arm would eventually follow. Next the user identifies three random points on the planar surface with the laser (Fig. 4(b)). After this, the user locates the target object with the laser and commands the execution of the autonomous trajectory (Fig. 4(c)). When the arm stops near the target (Fig. 4(d)), the user commands autonomous alignment with the planar surface (Fig. 4(e)). Now, as the arm is in a convenient configuration for grasping, the user makes fine adjustments in teleoperation to locate the gripper exactly over the target to grasp it. Grasping is achieved by keyboard commands given to an electronic hand. We have not considered the mechanics of grasping as it is out of the scope of the paper.

Next, the user commands the system to autonomously execute a path from the current location to the destination point while avoiding the obstacle. The path is executed in three linear segments shown in Fig. 4(f), 4(g) and 4(h). The first is from the current PUMA location to a point vertically above it so that the arm is clear of the tallest obstacle, the second segment is in the horizontal plane to a point vertically above the destination and the third is straight down to a point slightly above the destination. The orientation of the end-effector remains constant throughout the path. The initial and final transformation matrices for the segments are determined from forward kinematics and from the destination point coordinates. After the arm has traversed the path, the user makes fine movements to precisely locate the target over the destination and places it at the destination point.

The method using the laser aims to relieve the human from cognitive and physical load while performing an ADL since it requires only minimal user interaction. The human supervisor only issues high level commands while the trajectories are generated online and executed autonomously. Since the critical points located by the laser could be located anywhere in the environment, the laser assisted method is suitable for manipulation in unstructured environments.

\section{B. Grasping a Door Handle in an Opening a Door Task Execution}

Here the user begins by pointing the laser to three critical points on the door in teleoperation and to record their coordinates (Fig. 5(a)). Then, the user points the laser to the door handle and commands autonomous trajectory execution (Fig. 5(b)). Once near the handle (Fig. 5(c)), the system autonomously aligns the gripper with the door surface on the user command so that the door handle can be grasped from a convenient angle (Fig. 5(d)). The user then teleoperates to precisely locate the gripper over the handle to grasp the door handle. Opening a door is a complex task for robots to execute and there have been separate works on these [14], [15]. Our scope is limited to setting up the remote arm in a proper configuration to open the door.

\section{A Note on Special Cases}

In the ADLs demonstrated above the user points to three points on a planar surface to determine its orientation. However, for horizontal surfaces, only one point is sufficient as the vector necessary for alignment will only have a unit $z$ component and zero $x$ and $y$ components. Similarly, for vertical surfaces, pointing the laser to two points is sufficient. The third point can be computed by the system as having a random $\mathrm{z}$ coordinate and $x$ and $y$ coordinates of any of the two points recorded. Thus selecting three points is not necessary for most of the tasks. This makes the execution of the ADL easier and faster as the user has to teleoperate to select fewer points. 


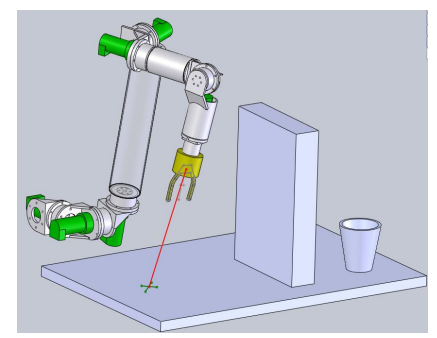

(a)

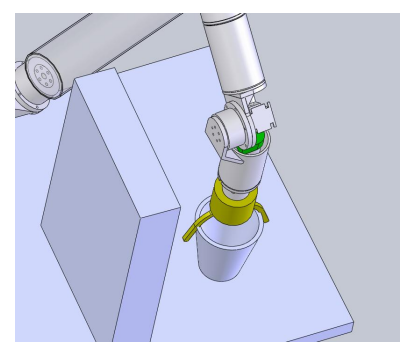

(e)

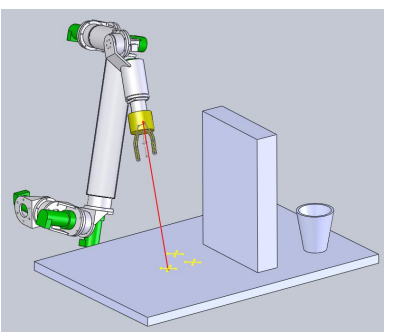

(b)

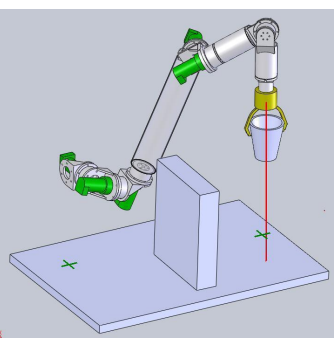

(f)

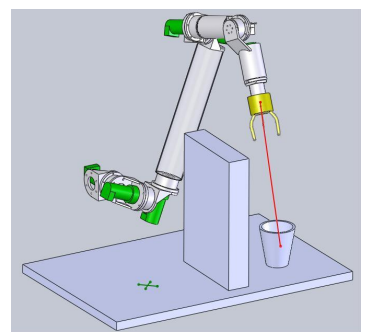

(c)

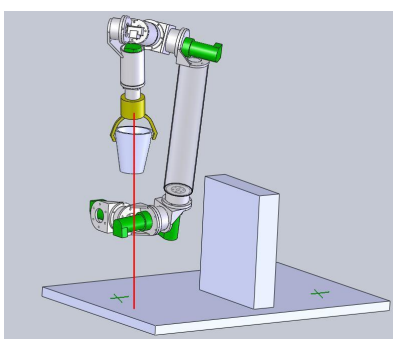

(g)

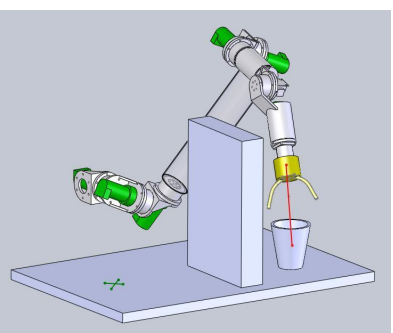

(d)

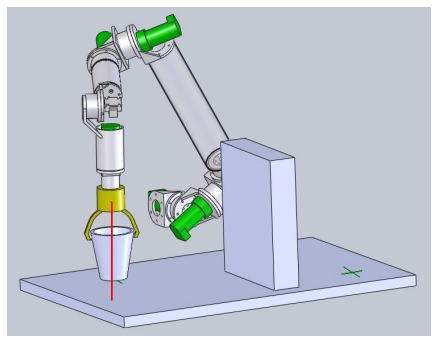

(h)

Fig. 4. Laser-based features in pick-and-place task execution: (a) Pointing to destination point for path planning (b) Pointing to platform points for surface orientation determination (c) Pointing to target (cup) for autonomous trajectory generation (d) End of autonomous trajectory (e) Autonomous end-effector alignment (f) Autonomous path end of first segment (g) Autonomous path end of second segment (h) Autonomous path end of third segment.

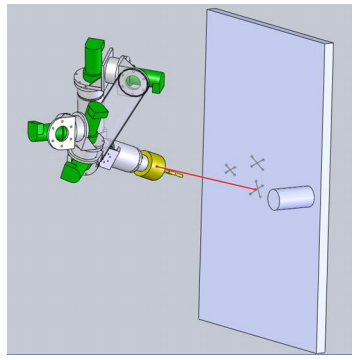

(a)

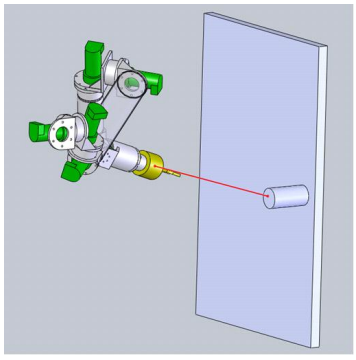

(b)

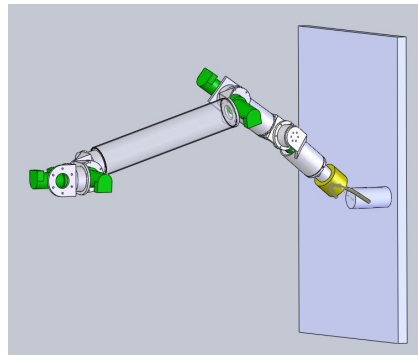

(c)

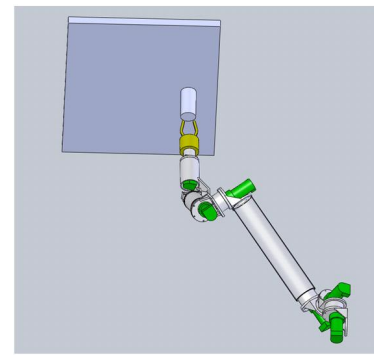

(d)

Fig. 5. Laser-based features in grasping a door handle in opening a door task execution: (a) Pointing to surface (door) points to determine surface orientation (b) Pointing to target (door handle) for autonomous trajectory generation (c) End of autonomous trajectory (d) Autonomous surface alignment for easy teleoperation.

\section{Evaluation Of The EfFectiveness Of Laser- ASSisted CONTROL METHOD}

\section{A. Experimental Test Bed}

Our test bed consists of a PUMA arm and an Omni haptic device (Fig. 6). A SICK DT60 laser range finder is mounted on the PUMA end effector (refer Fig. 1). The subjects could see the remote environment directly as the PUMA and Omni were close to each other. For applications in which the remote environment is further away, cameras can provide visual feedback. The PUMA and Omni were controlled on separate PCs communicating via Ethernet. The communication between PCs was at $200 \mathrm{~Hz}$ and controllers ran at $1000 \mathrm{~Hz}$. A real-time operating system, QNX, with a multithreaded programming architecture was used to control the PUMA. This provided real-time data processing and feedback capability for multiple sensors and actuators.
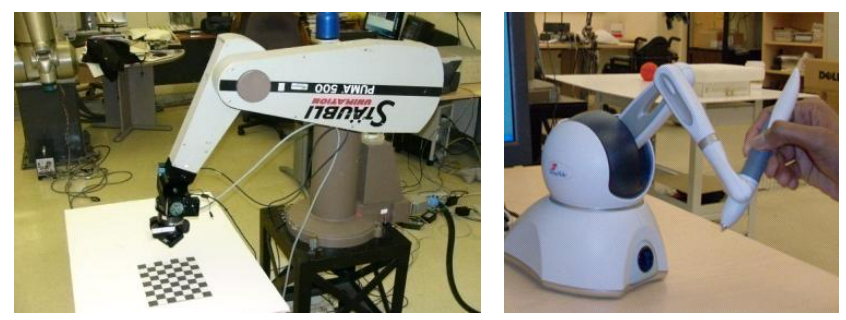

Fig. 6. Test bed of PUMA and Omni manipulators.

\section{B. Experimental Methodology and Set-up}

In order to evaluate the effectiveness of the laser-assisted method, human subject testing was carried out. Although the laser-based method is for assisting people with disabilities to perform ADLs, here we have tested healthy human subjects. We tested ten subjects, one female and nine males, ages 18 to 28 years. None of the subjects had any prior experience in teleoperating a robot arm. 
Each subject was asked to perform a pick-and-place task three times in each of the two modes: the unassisted teleoperation mode and the laser-assisted mode. In the unassisted mode, the complete task was executed solely by teleoperating the PUMA without any assistance except visual feedback. For each run the time taken to complete the task and the end effector transformation matrix was recorded at $1 \mathrm{msec}$ intervals. The user experience in executing the task was also recorded for each user. Before starting the tests, the subjects were given sufficient time to acclimatize with the system. In general, each subject was given 5 to 6 trials before testing.

The experimental set up is shown in Fig. 7. The cup is the target and is to be picked up from the source location shown and placed at the destination marked by the cross on the orange sticky. The folder simulates an obstacle and the white board is the platform. The task here is to start from a ready position, go towards the target, grasp it, and place it over the destination point while avoiding the obstacle.

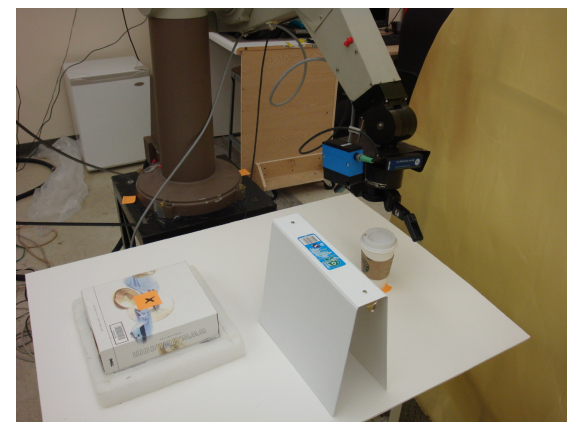

Fig. 7. Experimental set-up for pick-and-place task.

\section{RESUlts And Discussions}

The time that each user takes to execute the task in each mode and the effort expended in doing the task are the metrics used to evaluate the laser-assisted control method.

The average time to complete the task is shown in Fig. 8. We found that subjects took an average of $26.9 \%$ less time to complete the task in the laser-assisted mode than they did in the unassisted mode. An unpaired t-test, at $95 \%$ confidence level, shows that the time difference is statistically significant $(p<0.001)$.

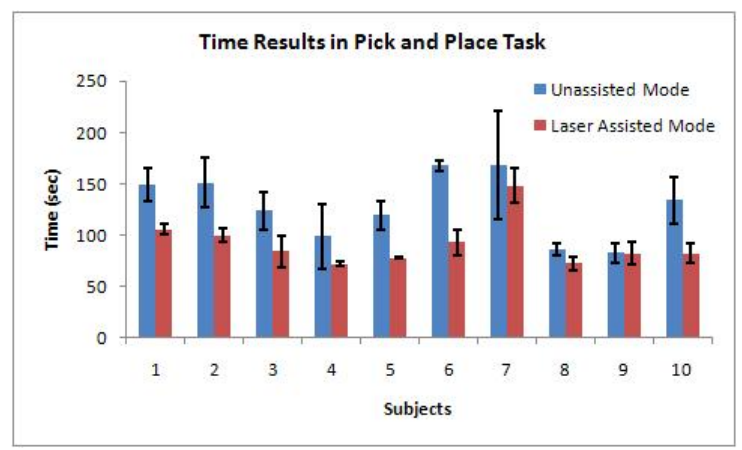

Fig. 8. Time plots in executing pick-and-place task.
The effort expended in executing the task was measured as the amount of movement of a subject's hand and arm while teleoperating the Omni in each of the two modes. The movement was broken up into distance traversed by the arm and rotation of the wrist. Total distance traversed by a subject's arm was determined by summing up the differential translation components of the PUMA transformation matrices recorded at each time step during task execution. As we have implemented position control in teleoperation, the amount of movement of the PUMA end-effector is the same as that of a subject's at the Omni. The total angle rotated by the subjects wrist during task execution was also determined by applying the equivalent angle-axis method to the differential rotation components of the transformation matrices recorded. Average values of arm distances and wrist angles per subject per mode for the three trials are shown in Fig. 9 and Fig. 10.

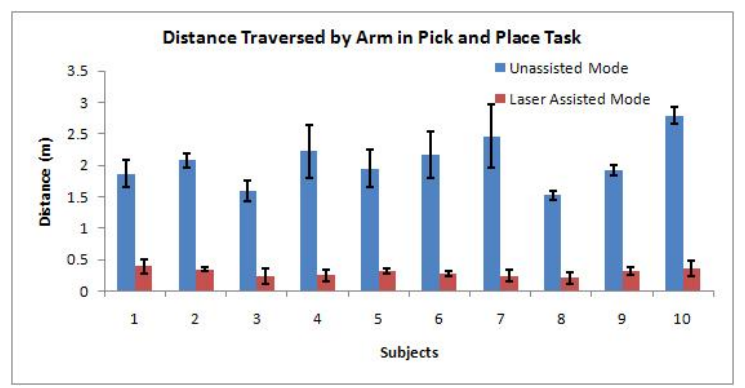

Fig. 9. Distance traversed by subjects arm in executing pick-and-place task

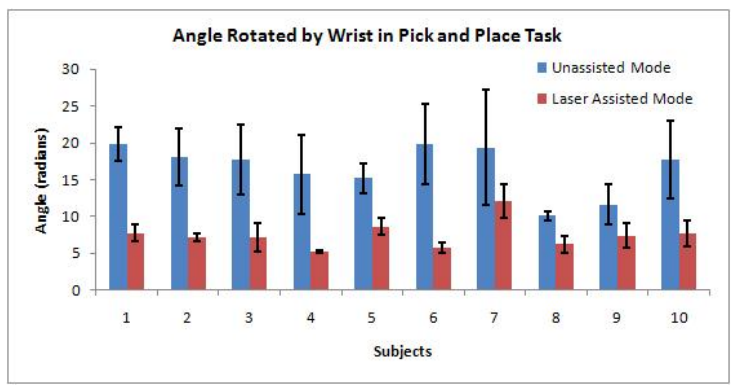

Fig. 10. Angle rotated by subjects wrist in executing pick-and-place task.

From these plots, we see that the subjects make larger movements with their hands and arms while executing the task in unassisted mode than they do in the laser-assisted mode. The average distance traveled is $85.4 \%$ less for the laser-assisted mode than the unassisted mode and $53.2 \%$ less for the total rotation. An unpaired t-test, at $95 \%$ confidence level, shows that both distances are statistically significant $(p<0.001)$. The large motions are physically tiring for the subjects and introduce fatigue in the hands and arms. These results become more significant when we consider that the methodology is going to be used to assist people with disabilities who have weak muscular strength.

An example 3D trajectory of the PUMA for one subject is shown in Fig. 11. We observe that the subjects had problems with maintaining a steady path and often deviated from 
the path in unassisted teleoperation mode. We also observe certain loops around the pick-up point. These are due to subjects repeatedly trying to orient the gripper properly so that it is in a convenient configuration for grasping. Orienting the arm properly was one of the most challenging activities the subjects faced. These plots make it clear that the subjects had tremendous difficulties in teleoperating the PUMA without any assistance, as is typical for many teleoperation environments. Similar plots were obtained for the other nine subjects.

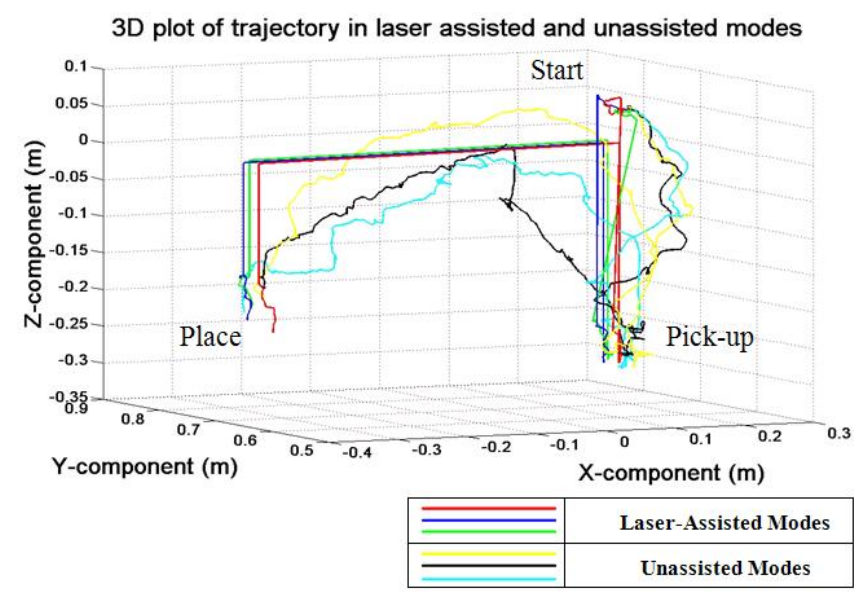

Fig. 11. 3D trajectory of remote robotic arm in laser-assisted and unassisted modes for trials on one subject.

From the user experience recorded, we learned that it was mentally challenging for the subjects to execute the task in the unassisted mode. Picking out points with the laser and commanding the robot to execute sub-tasks was much easier. They preferred the supervisory control mode in which they only generated high-level commands and the robot executed computationally intensive and low-level tasks. The subjects were relieved from being bothered about the complexities of the task.

\section{CONCLUSION}

Our hypothesis that the laser-assisted telerobotic control methodology of task execution would improve the task performance and reduce the physical and mental burden on the users in remote task execution has been validated by tests on healthy human subjects executing a pick-and-place task, a common ADL. With the results obtained, we believe that this telerobotic system would make it possible for persons with disabilities to execute ADLs with much greater ease. Next we intend to test the methodology on persons with disabilities. Our future work will include integrating vision algorithms to enable grasping the targets from multiple angles. Also, we intend to implement the laser-assisted telerobotic control concept on a wheelchair mounted robotic arm (WMRA) [16].

\section{REFERENCES}

[1] “Americans with disabilities: 2002," US Census Bureau, Tech. Rep., 2006.

[2] G. Bolmsjo, H. Neveryd, and H. Eftring, "Robotics in rehabilitation," IEEE Transactions on Rehabilitation Engineering, vol. 3, no. 1, pp. 77-83, 1995.

[3] S. Hayati and S. Venkataraman, "Design and implementation of a robot control system with traded and shared control capability," in IEEE International Conference on Robotics and Automation. USA: IEEE, 1989, pp. 1310-1315.

[4] Y. Yokokohji, A. Ogawa, H. Hasunuma, and T. Yoshikwa, "Operation modes for cooperating with autonomous functions in intelligent teleoperation systems," in IEEE International Conference on Robotics and Automation, vol. 3. USA: IEEE, 1993, pp. 510-515.

[5] T. Tarn, N. Xi, C. Guo, and Y. Wu, "Task-oriented human and machine co-operation in telerobotic systems," Annual Reviews in Control, vol. 20, pp. 173-178, 1996.

[6] L. Joly and C. Andriot, "Motion constraints to a force reflecting telerobot through real-time simulation of a virtual mechanism," in IEEE International Conference on Robotics and Automation, vol. 1. IEEE, 1995, pp. 357-362.

[7] P. Aigner and B. McCarragher, "Human integration into robot control utilizing potential fields," in IEEE International Conference on Robotics and Automation, vol. 1. IEEE, 1997, pp. 291-296.

[8] S. Everett and R. Dubey, "Human-machine cooperative telerobotics using uncertain sensor or model data," in IEEE International Conference on Robotics and Automation, vol. 2. IEEE, 1998, pp. 1615-1622.

[9] N. Pernalete, W. Yu, R. Dubey, and W. Moreno, "Development of a robotic haptic interface to assist the performance of vocational tasks by people with disability," in IEEE International Conference on Robotics and Automation, vol. 2. IEEE, 2002, pp. 1269-1274.

[10] W. Yu, R. Alqasemi, R. Dubey, and N. Pernalete, "Telemanipulation assistance based on motion intention recognition," in IEEE International Conference on Robotics and Automation. IEEE, 2005, pp. $1121-1126$.

[11] T. Hasegawa, T. Suehiro, and K. Takase, "A robot system for unstructured environments based on an environment model and manipulation skills," in IEEE International Conference on Robotics and Automation, vol. 1. IEEE, 1991, pp. 916-923.

[12] Y. Takahashi and M. Yashige, "Robotic manipulator operated by human interface with positioning control using laser pointer," in IEEE 26th Annual Conference of the Industrial Electronics Society, vol. 1. IEEE, 2000, pp. 608-613.

[13] H. Nguyen, C. Anderson, A. Trevor, A. Jain, Z. Xu, and C. Kemp, "El-e: An assistive robot that fetches objects from flat surfaces," in The Robotic Helpers Workshop at HRI'08, The Netherlands, 2008.

[14] A. Ng and A. Petrovskaya, "Probabilistic mobile manipulation in dynamic environments, with application to opening doors," in International Joint Conference on Artificial Intelligence. India: Morgan Kaufmann Publishers Inc., 2007, pp. 2178-2184.

[15] L. Peterson, D. Austin, and D. Kragic, "High-level control of a mobile manipulator for door opening," in Intelligent Robots and Systems, vol. 3. Japan: IEEE, 2000, pp. 2333-2338.

[16] K. Edwards, R. Alqasemi, and R. Dubey, "Design, construction and testing of a wheelchair-mounted robotic arm," in IEEE International Conference on Robotics and Automation. IEEE, 2006, pp. 3165-3170. 